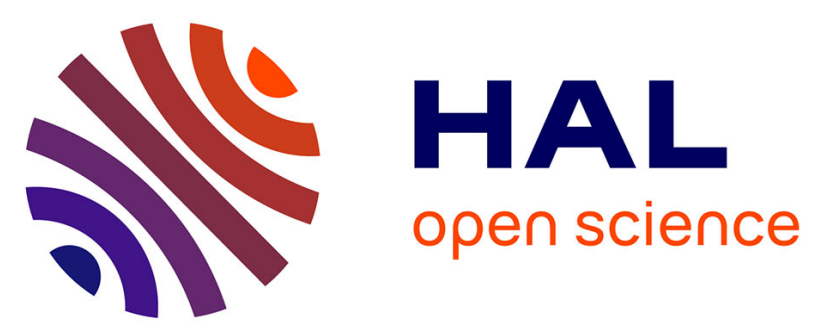

\title{
Innovative Low-Power Self-Nanoconfined Phase-Change Memory
}

Anna Lisa Serra, Gauthier Lefevre, Guillaume Bourgeois, Chiara Sabbione, Niccolo Castellani, Olga Cueto, Marie-Claire Cyrille, Mathieu Bernard, Julien Garrione, Nicolas Bernier, et al.

\section{To cite this version:}

Anna Lisa Serra, Gauthier Lefevre, Guillaume Bourgeois, Chiara Sabbione, Niccolo Castellani, et al.. Innovative Low-Power Self-Nanoconfined Phase-Change Memory. IEEE Transactions on Electron Devices, 2021, 68 (2), pp.535-540. 10.1109/TED.2020.3044267 . cea-03119677

\section{HAL Id: cea-03119677 https://hal-cea.archives-ouvertes.fr/cea-03119677}

Submitted on 25 Jan 2021

HAL is a multi-disciplinary open access archive for the deposit and dissemination of scientific research documents, whether they are published or not. The documents may come from teaching and research institutions in France or abroad, or from public or private research centers.
L'archive ouverte pluridisciplinaire HAL, est destinée au dépôt et à la diffusion de documents scientifiques de niveau recherche, publiés ou non, émanant des établissements d'enseignement et de recherche français ou étrangers, des laboratoires publics ou privés. 


\section{Innovative Low Power Self-Nano-Confined Phase- hhange Memory}

A. L. Serra, G. Lefevre, G. Bourgeois, C. Sabbione, N. Castellani, O. Cueto, M.-C. Cyrille, M. Bernard, J. Garrione, N. Bernier, C. Vallée, D. Sylvain, C. Charpin-Nicolle, G. Navarro*and E. Nowak

* Corresponding author: gabriele.navarro@cea.fr

Abstract-In this paper, we demonstrate at array level and in industrial like devices, the extreme scaling down to nanometric dimensions of the Phase-Change Memory technology thanks to an innovative Self-Nano-Confined PCM device (SNC PCM). We show how such solution based on an optimized GeN/GeSbTe stack enables programming down to $50 \mu \mathrm{A}$ and endurance up to more than $10^{8}$ cycles in $4 \mathrm{~kb}$ arrays, with the huge advantage of having no dependency on the critical lithography dimension used. We further demonstrate that the high thermal confinement achieved in such extremely confined PCM makes the engineering of the SET pulse becoming fundamental in order to assure a reduced SET resistance drift. Moreover, thanks to physico-chemical analyses and 3D TCAD electro-thermal simulations we demonstrate the Self-Nano-Confined phenomenon, revealing an effective scaling of the PCM down to around $12 \mathrm{~nm}$ and how it improves the thermal efficiency of the device thanks to a reduced current density and thermal stress in the system.

Index Terms-Low power Phase-Change Memory, Thermal confinement, Electro-thermal simulations.

\section{INTRODUCTION}

$\mathbf{P}$ HASE-CHANGE MEMORY (PCM) is a major candidate among the resistive Non-Volatile Memory (NVM) technologies. Featuring properties of both DRAM and Flash, its advanced state in terms of development and industrialization in Storage Class Memory applications [1] and in embedded PCM-based microcontrollers for automotive applications [2] makes it an undeniable breakthrough in the present storage scenario. Even if phase-change mechanism with current in the microampere range has already been demonstrated for nanometric analytical devices [3], current reduction remains

This work was partially funded by European commission, French State and Auvergne-Rhône Alpes region through ECSEL project WAKEMEUP and French Nano2022 program.

A. L. Serra, G. Bourgeois, C. Sabbione, N. Castellani, O. Cueto, M. C. Cyrille, M. Bernard, J.Garrione, N. Bernier, C. Charpin, G. Navarro and E. Nowak are with Univ. Grenoble Alpes, F-38000 Grenoble, France CEA, LETI, MINATEC Campus, F-38054 Grenoble, France (e-mail: annalisa.serra@cea.fr, guillaume.bourgeoise@cea.fr,_chiara.sabbione@cea.fr, niccolo.castellani@cea.fr, olga.cueto@cea.fr, marie-claire.cyrille@cea.fr, mathieu.bernard@cea.fr,_julien.garrione@cea.fr,_nicolas.bernier@cea.fr, christelle.charpin@cea.fr, etienne.nowak@cea.fr, corresponding author: gabriele.navarro@cea.fr).

G. Lefevre, C. Vallée and D. Sylvain are with CNRS-LTM Laboratoire des Technologies de la Microélectronique, 38054 Grenoble, France (e-mail: gauthier.lefevre@cea.fr, Sylvain.DAVID@cea.fr and christophe.vallee@cea.fr). the main requirement for PCM products to address next generation of NVM low power applications. Several solutions have been proposed so far, such as: a) aggressive lithography and architecture solutions [4], b) more or less complex material engineering [5], c) interfaces and thermal efficiency improvement [6]. Despite the promising results, these solutions still raise doubts about the technology cost, its scalability in industrial devices, the material evolution control during Back-End-Of-Lines (BEOL) fabrication steps, and the material degradation along cycling especially in scaled PCM. PCM stacks based on oxide interfaces have been proposed in order to reduce the programming current [7], but without scaling capability demonstration and a possible detrimental oxygen impact on the integrity of the chalcogenide material [8] [9]. In this work, we propose a Self-Nano-Confined PCM (SNC PCM) solution based on an optimized oxide-free GeN/GeSbTe stack integrated in state of the art "Wall" PCM (Fig. 1). The device features high stability and integrity even during BEOL fabrication (one hour at $400{ }^{\circ} \mathrm{C}$ ) and a reliable low voltage initialization step. We show RESET current $\left(I_{R}\right)$ reduction down to $50 \mu \mathrm{A}$, without any dependence on the device size, suggesting the existence of a self-nano-confined (SNC) mechanism responsible for a higher thermal efficiency of the device. This result is very encouraging since previous work performed on PCM arrays presented a minimum RESET current of $100 \mu \mathrm{A}$ achieved either with not scaled devices (critical dimension

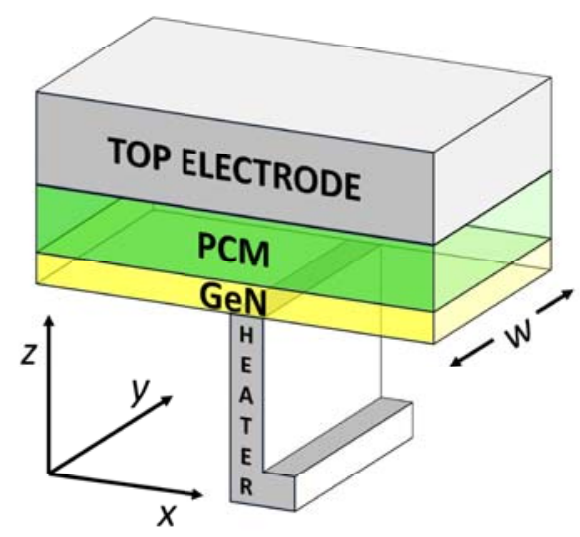

Fig. 1. Simplified schematic of the as fabricated SNC PCM "Wall" device based on a GeN/GeSbTe stack. 


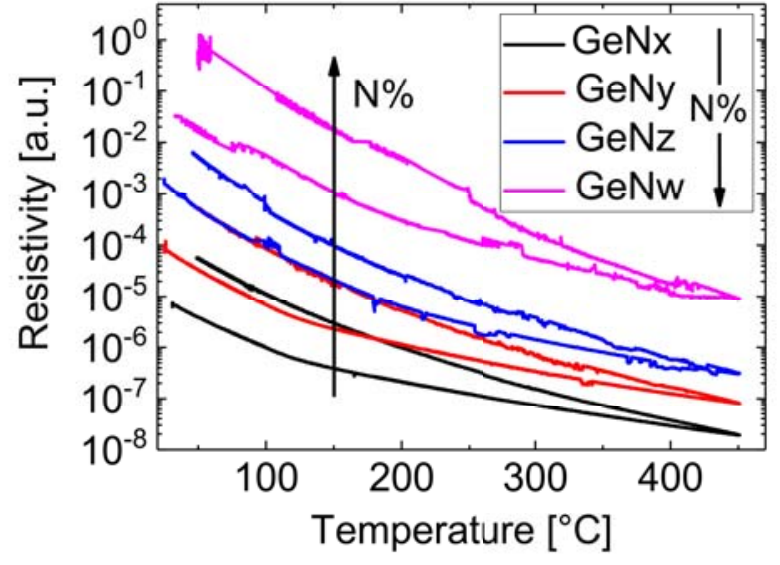

Fig. 2. $\mathrm{N}$ concentration tuning in $\mathrm{GeN}$ layer. The higher the $\mathrm{N}$ concentration from $x$ to $\mathrm{w}$, the higher the resistivity.

CD equal to $200 \mathrm{~nm}$ ) [10] or extremely scaled (CD equal to $20 \mathrm{~nm}$ ) [11] but expensive because of complex fabrication. The results presented in this work are supported by detailed Transmission Electron Microscopy (TEM) images supported by Energy-dispersive X-ray spectroscopy (EDX) analyses and 3D TCAD electro-thermal simulations, revealing an effective scaling of our PCM down to about $12 \mathrm{~nm}$. The reduced current density and thermal stress in SNC PCM enable an endurance up to more than $10^{8}$ cycles. Finally, we demonstrate the need for an optimized SET operation in extremely confined PCM device featuring high thermal confinement.

\section{DEVICE FABRICATION AND ELECTRICAL CHARACTERIZATION}

\section{A. GeN layer analysis and device integration}

The SNC PCM functionality is based on the soft breakdown of the GeN layer performed as a first step (i.e. initialization) on as fabricated devices. The reliability of such electrical initialization depends on the layer uniformity, its resistivity and the tuning of the electric field required. We engineered the GeN layer by exploiting different $\mathrm{N}$ contents and thicknesses in order to tune its resistivity, the electric field needed to initialize the layer, and its stability at high temperature targeting the BEOL compatibility and best device performances. The resistivity as a function of temperature measured by the fourpoint-probe method is reported in Fig. 2 and it shows the increase of the resistivity with $\mathrm{N}$ content. Intermediate $\mathrm{N}$ content introduced in $\mathrm{GeN}_{\mathrm{y}}$ layer was finally chosen for integration in electrical devices, featuring the right compromise in terms of resistivity even after annealing at $450{ }^{\circ} \mathrm{C}$.

The GeN/GeSbTe optimized stack was integrated by magnetron sputtering in $4 \mathrm{~kb}$ arrays of state of the art "Wall" based PCM devices fabricated in the BEOL of the fabrication of LETI Memory Advanced Demonstrator (MAD) based on $130 \mathrm{~nm}$ CMOS technology, for statistical electrical parameters evaluation. Fig. 1 reports a 3D simplified description of the as fabricated cell. The devices have a nominal width $(w)$ ranging from $50 \mathrm{~nm}$ up to $300 \mathrm{~nm}$.

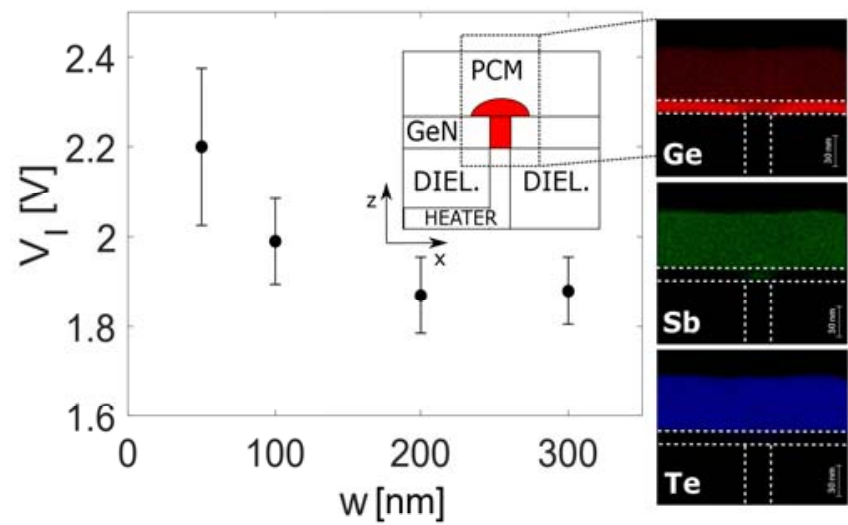

Fig. 3. Mean and $\pm 1 \sigma$ values for the initialization voltage $\left(V_{1}\right)$ measured in arrays as a function of $w$ (left). TEM/EDX ( $x z$ plane) analysis of a device initialized at $1.2 \mathrm{~mA}$, showing the SNC phenomenon and the absence of elemental segregation (right). The inset schematizes the shape of the active volume of the SNC PCM.

\section{B. Electrical characterization of $4 \mathrm{~kb} P C M$ arrays}

Preliminary tests are performed on $4 \mathrm{~kb}$ arrays to confirm the GeN layer integrity and the device functionality. The initialization voltage $\left(\mathrm{V}_{\mathrm{I}}\right)$, which is used to supply the bit-line of the arrays, shows a slight evolution and a low variability with respect to $w$ (Fig. 3). The slight decrease of $\mathrm{V}_{\mathrm{I}}$ observed increasing $w$ is associated to the known phenomena of materials dielectric strength change with material shape and size [12]. The inset in Fig. 3 describes the initialization mechanism, showing in red the region involved in the programming of the cell after the initialization procedure (i.e. the active volume). The TEM/EDX analysis after initialization reported in the same figure confirms the diffusion of $\mathrm{Ge}, \mathrm{Sb}$ and Te in the GeN constriction with the formation of a GeSbTe confined region without facing any material segregation. This

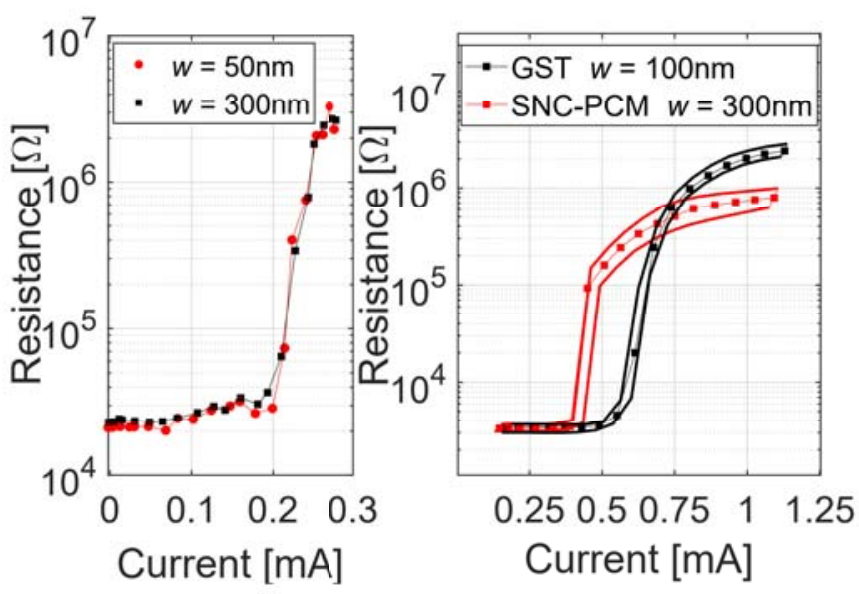

Fig. 4. (Left) $1 T 1 R$ RI characteristics for $50 \mathrm{~nm}$ and $300 \mathrm{~nm}$ devices obtained with $10 \mathrm{~ns} / 300 \mathrm{~ns} / 10 \mathrm{~ns}$ staircase pulses. Size-independency of SNC mechanism is here verified with $\mathrm{I}_{R}$ of about $250 \mu \mathrm{A}$. (Right) Median and $\pm 1 \sigma$ values for RI data comparing the behavior of a SNC PCM (with $w$ equal to $300 \mathrm{~nm}$ ) with respect to standard PCM ( $w$ equal to $100 \mathrm{~nm}$ ) based on $\mathrm{Ge}_{2} \mathrm{Sb}_{2} \mathrm{Te}_{5}$ (GST). The SNC PCM is $3 x$ larger but it assures the same programming current of GST with a steeper transition. 


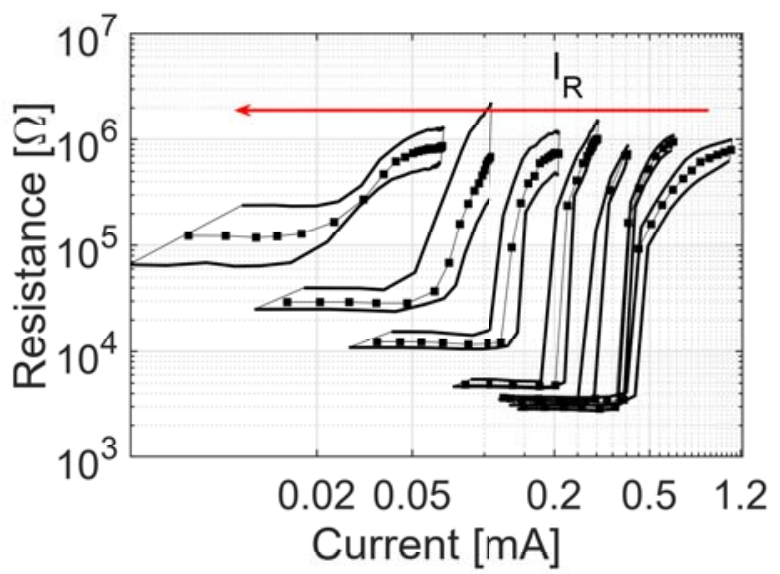

Fig. 5. Median and $\pm 1 \sigma$ values for the RI characteristics in log-log scale obtained in $4 \mathrm{~kb}$ arrays at different $\mathrm{I}_{\mathrm{R}} \mathrm{S}$. SNC PCM devices demonstrate a programming current reduction down to $50 \mu \mathrm{A}$.

support the idea that the SNC mechanism is taking place in our devices. Resistance vs Current (RI) characteristics of 1T1R (i.e. 1 transistor/1 resistor) devices demonstrate that the SNC PCM mechanism is independent from the device size $w$ (Fig. 4 (left)), showing identical RI for device widths of $50 \mathrm{~nm}$ and $300 \mathrm{~nm}$ when initialized with the same current. The main advantage is that the mechanism can be tuned by controlling the bit line current that flows in the device during the initialization step, therefore it has the role to define both the $\mathrm{CD}$ and the RESET current of the devices. The initialized devices are firstly programmed in the SET state with a squared pulse "SP" (i.e. rise/width/fall time equal to $10 \mathrm{~ns} / 300 \mathrm{~ns} / 10 \mathrm{~ns})$. The gradual resistance increase towards the RESET state is obtained with a staircase up sequence of SPs of increasing amplitude (with steps of $0.1 \mathrm{~V}$ ). Furthermore, in Fig. 4 (right) we compare the RI characteristics obtained in $4 \mathrm{~kb}$ arrays for SNC PCM with respect to the standard GST cell highlighting the same programming current in both the devices $(1.2 \mathrm{~mA})$ only if the $w$ in a standard cell is reduced by a scale factor of three. The steeper SET-RESET transition in SNC PCM is a particular evidence of the confined structure achieved thanks to the initialization step. By tuning the current in the initialization, it was possible to obtain seven different RI characteristics (Fig. 5) and show that the SNC mechanism can take place down to RESET current of $50 \mu \mathrm{A}$. The SET resistance increase at low $I_{R}$ is a clear evidence of the smaller diameter achieved during the initialization process.

By taking into account the inverse proportionality between the dynamic resistance of the devices $\left(\mathrm{R}_{\mathrm{ON}}\right)$ and the area of the initialized confined region of the GeN layer, it is possible to consider:

$$
I_{R} \cdot R_{O N} \propto J_{R}
$$

where $I_{R}$ is the RESET current and $J_{R}$ is the associated current density. The $I_{R}$ as a function of the inverse of $R_{O N}$ is reported in Fig. 6 which is useful to follow the evolution of $J_{R}$ in the different device populations. The data can be divided in two regions each one characterized by a different slope $\left(s_{1}\right.$ and $\left.s_{2}\right)$. The lower value of $s_{1}$ is correlated to the $J_{R}$ reduction in devices belonging to the Lower Currents

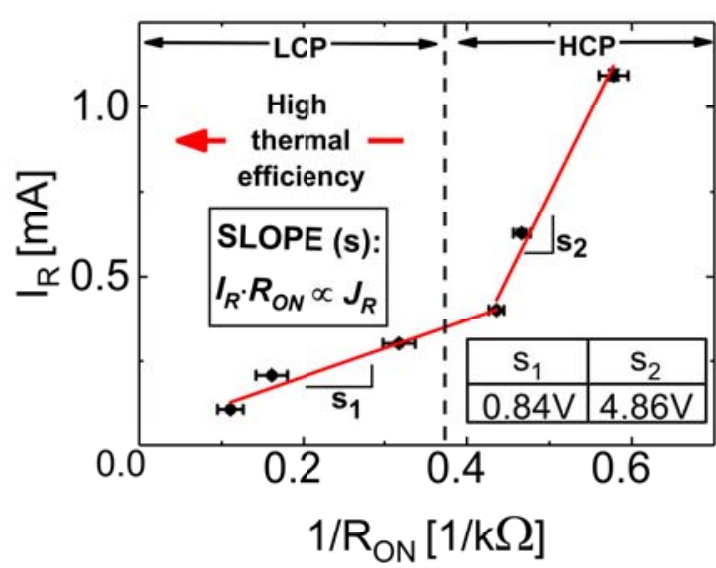

Fig. 6. Median and $\pm 1 \sigma$ values for the RESET current $\left(I_{R}\right)$ as a function of the inverse of the dynamic device resistance $\left(R_{O N}\right)$ in $4 \mathrm{~kb}$ arrays. The different slopes evidence the lower current density $\left(J_{R}\right)$ needed to program devices initialized with low $I_{R}(L C P)$ with respect to the ones for which a high $I_{R}$ is used (HCP). A lower slope in LCP population is highlighting a better thermal efficiency of the programming operation in such SNC PCM devices.

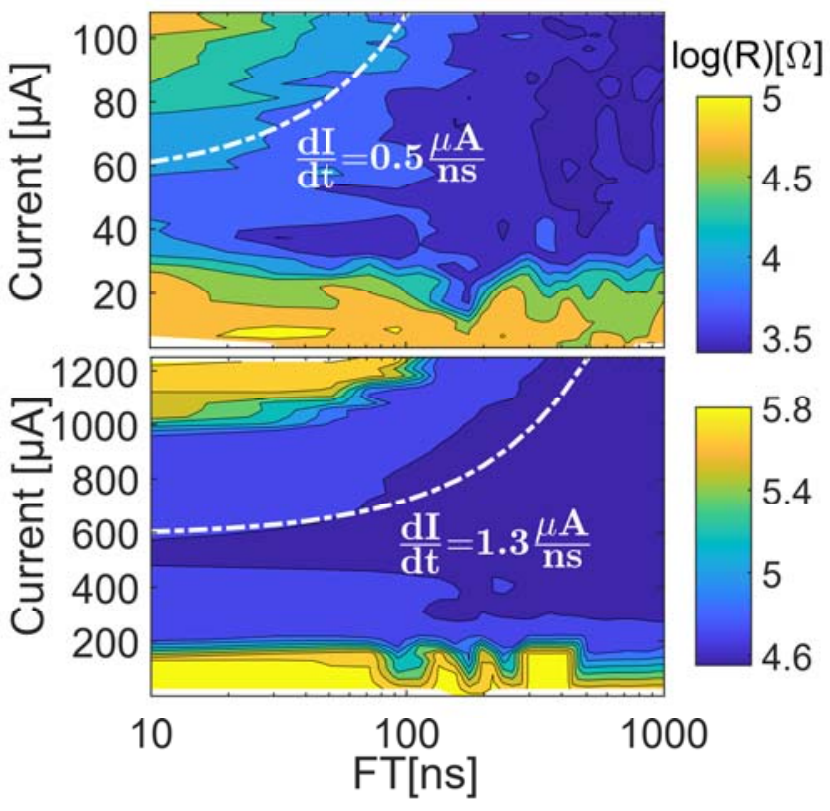

Fig. 7. SET cartographies showing the SET resistance evolution as a function of the pulse fall time (FT) (x-axis) and of the pulse current ( $y$-axis). The results are reported for a 1T1R device initialized at $100 \mu \mathrm{A}$ (top) and $1.2 \mathrm{~mA}$ (bottom). The slope $\boldsymbol{d I} / \boldsymbol{d t}$ needed to reliably SET the cell (i.e. equal to $d I / d t_{S E T}$ used to achieve a $3 \times$ RESET resistance lowering) is more than two times lower in SNC PCM owning to LCP.

Population (LCP) leading to a better thermal efficiency with respect to the devices in the High Currents Population (HCP).

The analysis on the SET programming speed is reported in the SET cartographies of Fig. 7 performed on 1T1R devices from $\operatorname{LCP}\left(\mathrm{I}_{\mathrm{R}}\right.$ equal to $\left.100 \mu \mathrm{A}\right)$ and $\mathrm{HCP}\left(\mathrm{I}_{\mathrm{R}}\right.$ equal to $\left.1.2 \mathrm{~mA}\right)$. The test is done by acquiring the resistance achieved in the cell after the application of a RESET pulse followed by a SET pulse (with a fixed pulse width of $300 \mathrm{~ns}$, and increasing 


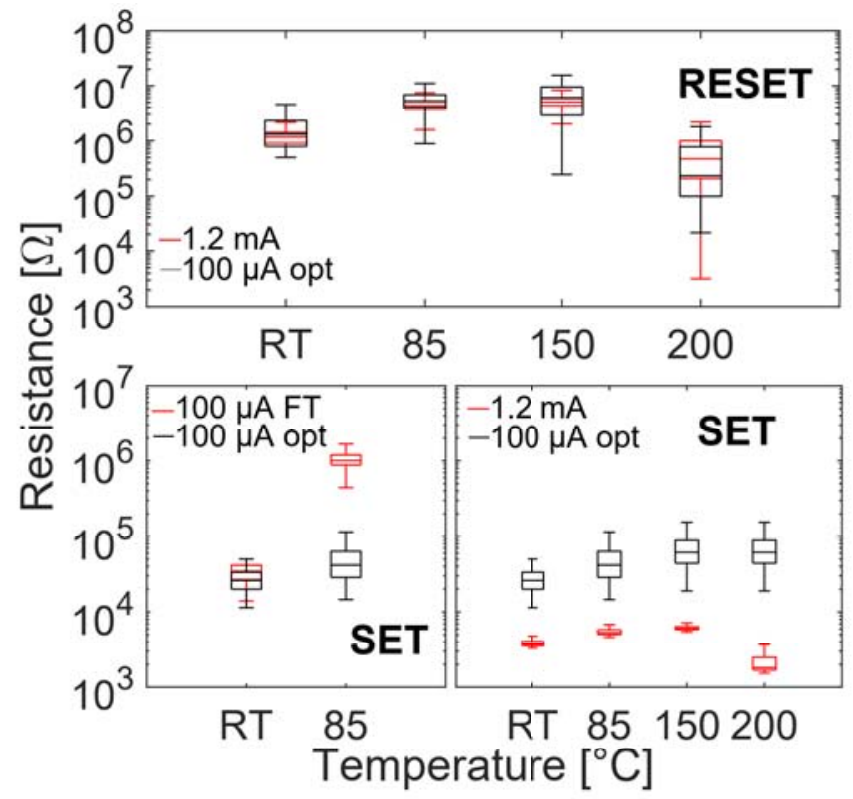

Fig. 8. RESET (top) and SET (bottom) data retention performed by isochronal annealing of one hour at increasing temperatures on $4 \mathrm{~kb}$ arrays with $\mathrm{I}_{\mathrm{R}}$ of $1.2 \mathrm{~mA}$ and $100 \mu \mathrm{A}$. The RESET retention performances are comparable for the two SNC PCMs. SET stability is ensured by SET-opt protocol, since a standard FT strategy leads to a high resistance drift already at $85{ }^{\circ} \mathrm{C}$.

fall time and amplitude). The dashed lines in the figure represent a linear fit used to evaluate the current over time ratio $(d I / d t)$ needed during the pulse fall time to reliably SET the cell (i.e. to decrease the resistance value threefold with respect to the RESET state). The optimal $d I / d t$ (i.e. $d I / d t_{S E T}$ ) ratio is more than two times lower in devices belonging from LCP, revealing the need of an optimized SET pulse to fulfill the specific requirement. Data retention tests on RESET and SET states were performed for the two populations LCP and HCP under investigation. Both the standard SET and the optimized SET were compared in terms of retention. The RESET state (Fig. 8) shows a comparable behavior for both the high and low initialization currents, with a resistance drift at $85{ }^{\circ} \mathrm{C}$ and a beginning of recrystallization at $200{ }^{\circ} \mathrm{C}$, confirming the preservation of the material properties even in nm-scale SNC PCM. The comparison between the standard (SET-FT) and optimized SET (SET-opt) shows that the optimized SET protocol helps reducing the resistance drift faced as soon as the temperature is increased, certifying the higher crystalline homogeneity obtained in the confined region of the SNC PCM device with respect to the standard pulse. The result achieved with the optimized pulse is also evidenced in the subthreshold conduction analysis (Fig. 9). In particular, the LCP device programmed with a SET-opt procedure reveals a reliable ohmic behavior, comparable with the device initialized at higher current and programmed with a standard pulse. On the contrary, the $100 \mu \mathrm{A}$ device programmed in SET state with a standard pulse shows an exponential conduction, that we can correlate with a non-uniform crystalline or partially amorphous structure having trap-dominated conduction [13].

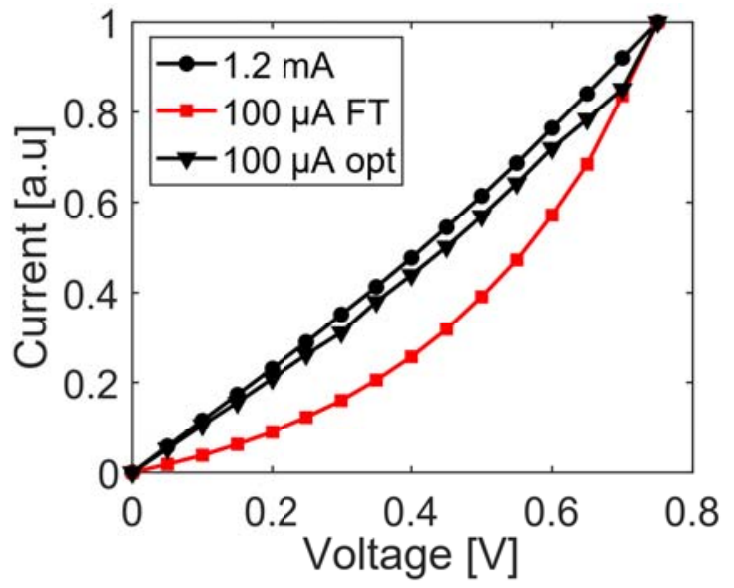

Fig. 9. Normalized subthreshold IV curves for 1T1R devices programmed at $1.2 \mathrm{~mA}(\mathrm{HCP})$ and $100 \mu \mathrm{A}(\mathrm{LCP})$. The ohmic behavior observed in HCP devices is achieved in LCP ones only with optimized SET protocol.
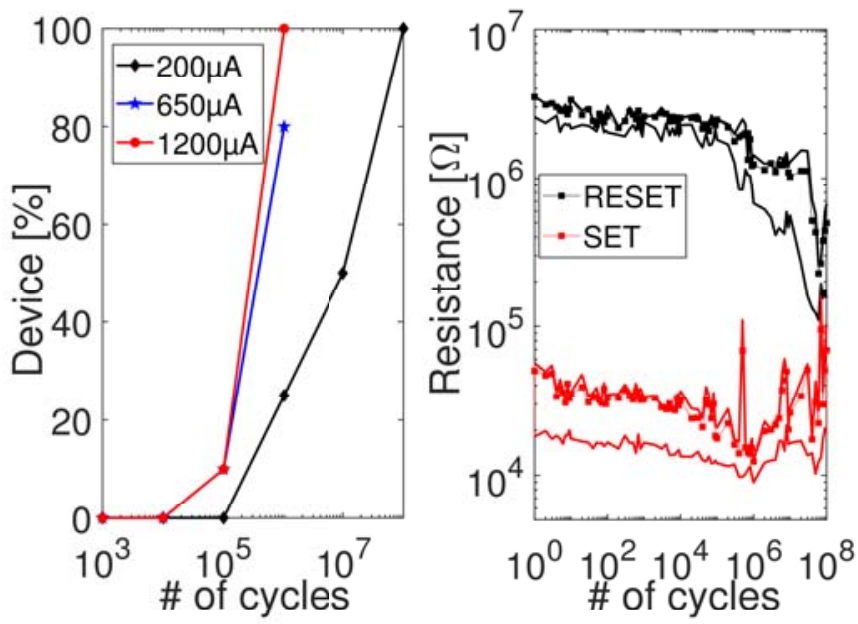

Fig. 10. Device endurance analysis showing the statistics for the starting of the resistance window degradation, detected as a $3 x$ reduction of the starting RESET resistance, for three different values of initialization current $I_{R}$. Ten devices has been tested for $650 \mu \mathrm{A}$ and $1200 \mu \mathrm{A}$ population and four for the $200 \mu \mathrm{A}$ due to their best endurance performance up to $10^{8}$ cycles.

The endurance is performed to study the material evolution along cycling for three populations ranging from LCP to HCP (i.e. initialized at $200 \mu \mathrm{A}, 650 \mu \mathrm{A}$ and $1200 \mu \mathrm{A}$ ). The comparison is made detecting when the resistance window starts to be reduced in particular because of the RESET resistance decreasing. The percentage of devices facing such starting of degradation is reported as a function of cycling in Fig. 10. It shows that the degradation starts at $10^{6}$ cycles for devices with $\mathrm{I}_{\mathrm{R}}$ equal to $650 \mu \mathrm{A}$ and $1200 \mu \mathrm{A}(\mathrm{HCP})$, while in SNC PCM featuring the lower $I_{R}$ (LCP) the same order of degradation is observed only at $10^{8}$ cycles. To be noted that in all the tests performed, all the devices kept at least a resistance window of one order of magnitude after $10^{6}$ cycles. Furthermore, the measured degradation results to be accelerated by the use of the same pulses (RESET: $10 \mathrm{~ns} / 300 \mathrm{~ns} / 10 \mathrm{~ns}$ and SET: $10 \mathrm{~ns} / 300 \mathrm{~ns} / 10 \mu \mathrm{s}$ ) for the three populations at 

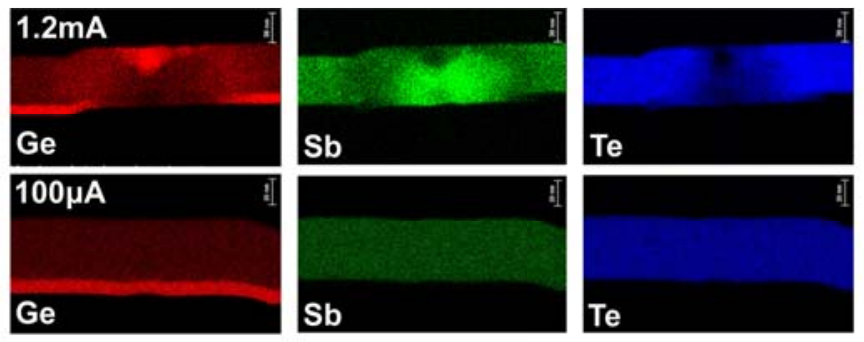

Fig. 11. TEM/EDX Ge, $\mathrm{Sb}$ and Te cartographies for SNC PCM cycled $10^{6}$ times, respectively at $1.2 \mathrm{~mA}$ (top) and $100 \mu \mathrm{A}$ (bottom). At $1.2 \mathrm{~mA}$ the GeN layer degrades along cycling with strong elemental segregation. On the contrary, $100 \mu \mathrm{A}$ device does not show signs of degradation (programmed region not visible because of its $\mathrm{nm}$ dimension).

the maximum $I_{R}$. Therefore, this result is even more representative of the high reliability of SNC PCM devices, that could feature higher endurance if benefiting of optimized or intelligent cycling algorithms [14]. The resistance evolution along cycling for a RESET current of $200 \mu \mathrm{A}$ is reported in Fig. 10, where it is possible to evidence the type of RESET lowering we observed independently from the initialization current applied (i.e. the RESET lowering mechanism is the same but it happens at a different number of cycles). The device degradation under cycling is studied by the TEM/EDX cartographies (Fig. 11), performed on devices cycled up to $10^{8}$ cycles for both $\mathrm{I}_{\mathrm{R}}$ of $1.2 \mathrm{~mA}$ (top) and of $100 \mu \mathrm{A}$ (bottom). The analysis confirms the higher level of segregation triggered after $10^{6}$ cycles in HCP devices, while no sign of degradation appears in LCP where the GeN layer results to be still uniform after cycling. To be noted that the signal reduction due to the presence of the filament is quite small in the device programmed at $100 \mu \mathrm{A}$ because of the signal integration along the width of the lamella used for the analysis. The SNC PCM stability was verified after a BEOL like thermal budget of one hour at $400{ }^{\circ} \mathrm{C}$. By increasing the annealing time, the GeN layer faces a structural reorganization [15], which results in the $V_{I}$ lowering (Fig. 12). Nevertheless, the TEM/EDX image (inset) and the SET/RESET distribution for $200 \mu \mathrm{A}$ cells show that the GeN layer integrity is kept even after an annealing of one hour at $400{ }^{\circ} \mathrm{C}$ and the devices can be programmed with a similar resistance window.

\section{ELECTRO-THERMAL SIMULATIONS}

3D electro-thermal simulation were performed in COMSOL Multiphysics [16] and run on SNC PCM with different critical dimensions. Fig. 13 represents the temperature profile reached in the PCM at the same current density $\mathrm{J}$ for devices having a $\mathrm{CD}$ of $12.5 \mathrm{~nm}$ (representative of the LCP) and of $37.5 \mathrm{~nm}$ (representative of the $\mathrm{HCP}$ ). It is possible to observe a higher maximum temperature in devices with the smaller $\mathrm{CD}$, highlighting the higher thermal efficiency of SNC PCM featuring lower $I_{R}$. In Fig. 14 the results of the simulations for the current density at the melting of the PCM material, shows its increasing moving towards a higher $\mathrm{CD}$, in accordance with experimental results previously reported in Fig. 6. Furthermore, it is shown that a higher $\mathrm{J}$ leads to
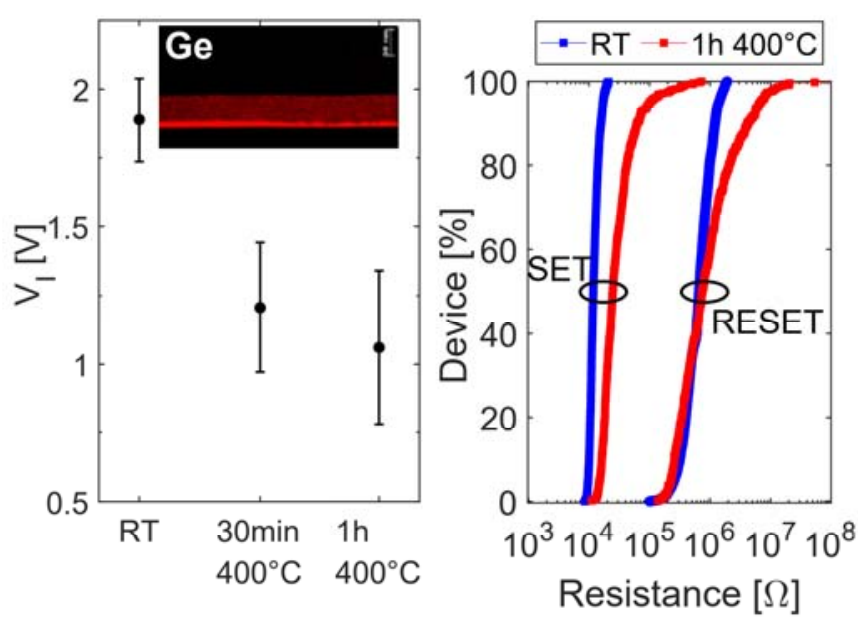

Fig. 12. Results of BEOL like thermal stress at $400{ }^{\circ} \mathrm{C} . \mathrm{GeN}_{\mathrm{y}}$ layer integrity is confirmed by TEM/EDX analysis (inset). $V_{1}$ is lowered at around $1 \mathrm{~V}$ after annealing. SNC mechanism is preserved after BEOL, as demonstrated in SET/RESET distributions for $I_{R}$ equal to $200 \mu \mathrm{A}$, compared before and after annealing.
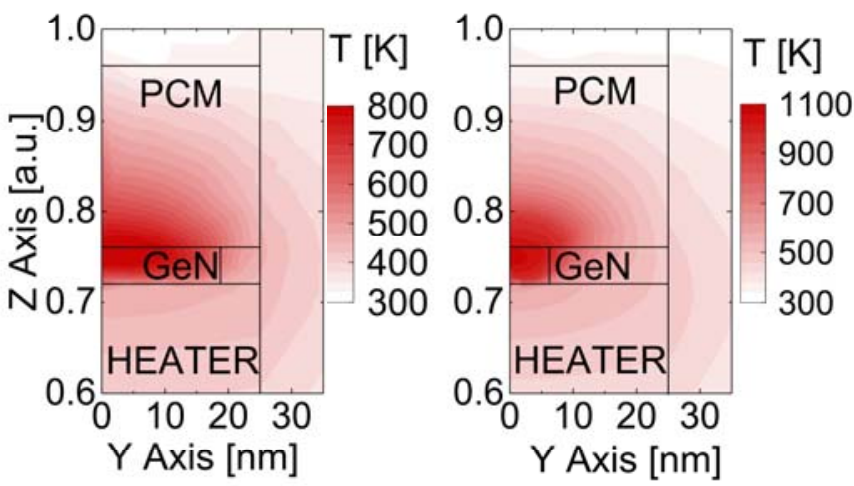

Fig. 13. Temperature profile simulated for SNC PCM with CD of $12.5 \mathrm{~nm}$ (right) and $37.5 \mathrm{~nm}$ (left) using the same current density J. Higher temperature is achieved in smaller SNC PCM thanks to its higher thermal efficiency.

a higher maximum temperature, which is likely responsible for a faster degradation of the material as observed in the endurance tests and TEM/EDX image (Fig. 10 and Fig. 11) in the devices initialized with high $\mathrm{I}_{\mathrm{R}}$. Moreover, the analysis performed on the temperature uniformity inside the constriction generated in the GeN layer, shows a lower temperature gradient $d T / d z$ (i.e. higher uniformity) in devices with small CD (Fig. 15). In general,

$$
\Delta T=R_{t h} R_{O N} I^{2}
$$

where $\Delta T$ is the temperature increase in a device having a specific thermal resistance $R_{t h}$ at a given programming current $I$. Hence:

$$
\frac{d T}{d t} \propto \frac{d I}{d t}
$$

It means that during the SET operation, in order to reliably program the device, the current over time rate reduction during the fall time of the SET pulse (i.e. $d I / d t_{S E T}$ measured in Fig. 7) depends on the following relation: 


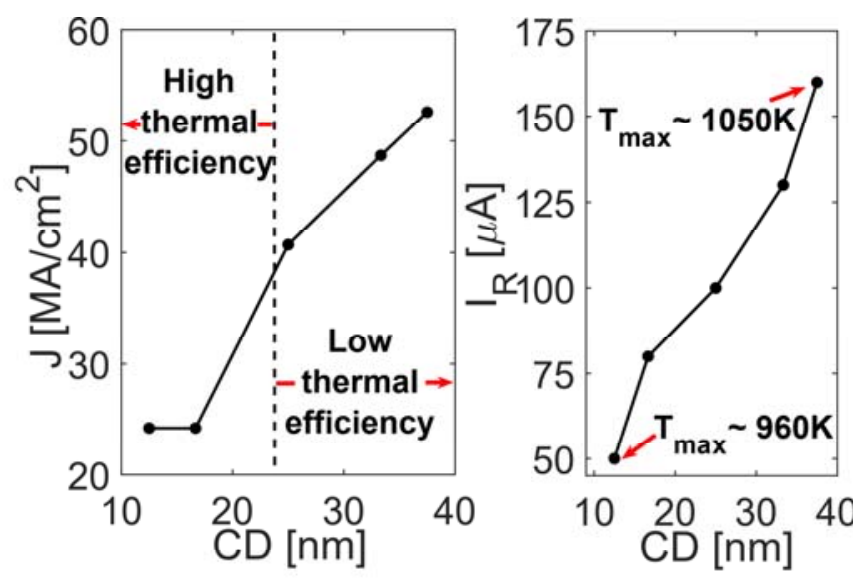

Fig. 14. Simulated $\mathrm{J}$ needed to achieve the melting of the material as a function of $C D$ (left). $J$ is lower in small $C D$ (i.e. higher thermal efficiency) in accordance with Fig. 6 . $I_{R}$ extracted from simulations as a function of $C D$ (right), shows that the maximum temperature ( $T_{\max }$ ) achieved during RESET operation is lower for smaller CD. The CD extracted at a $I_{R}$ of $50 \mu A$ is of around $12 \mathrm{~nm}$, that is the expected CD in the devices initialized at such low current in Fig. 5.

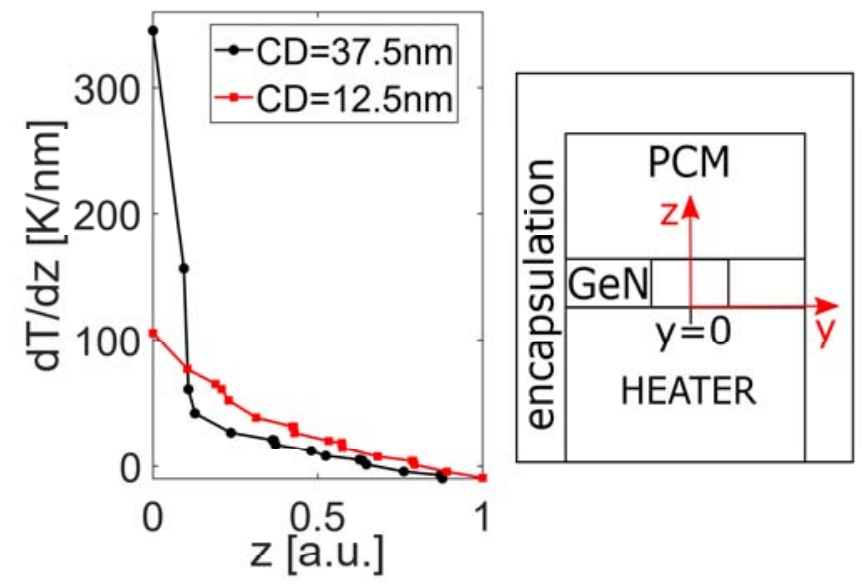

Fig. 15. Simulated temperature gradient along $z$-axis $(d T / d z)$ in the confined region of SNC PCM (at $y=0)$ for two devices featuring different $\mathrm{CD}$ size. A higher temperature homogeneity (i.e. lower $\boldsymbol{d T} / \boldsymbol{d z}$ ) is achieved in highly scaled confined SNC PCM featuring small CD.

$$
\frac{d I}{d t}_{S E T} \propto v_{g} \frac{d T}{d z}
$$

where $v_{\mathrm{g}}$ is the material crystalline growth speed. Therefore, the improved temperature uniformity in smaller CD observed in our simulations (Fig. 15) explains the lower $d I / d t_{S E T}$ measured in devices initialized at low current and featuring small CDs. This result supports the need for an optimized SET protocol in extremely confined devices, like in our SNC PCM.

\section{CONCLUSION}

This work demonstrates for the first time at array level and in industrial like devices, the extreme scalability down to nm scale of PCM technology thanks to an innovative and lithography-free SNC PCM device. We show the possibility to customize the programming condition of our device independently from the size and down to a programming current of $50 \mu \mathrm{A}$. We demonstrated the need for a lower current density to program the cells initialized at currents lower than $400 \mu \mathrm{A}$. Thanks to 3D TCAD electrothermal simulations we highlighted that such lower current density is related to a better thermal confinement of the cell: the maximum temperature was shown to decrease with the device CD in III. Endurance up to more than $10^{8}$ cycles is presented for $\mathrm{nm}$ scaled SNC PCM devices. This result is supported by TEM/EDX anlyses showing that $10^{6}$ cycles do not impact the GeN interfacial layer for low CD devices. Furthermore, the SET programming cartographies show the need for a SET pulse protocol optimization for highly scaled SNC PCM, which assures a reduced SET resistance drift and a data retention comparable with the devices initialized at higher current. The SNC PCM solution presented shows also a perfect integrity after BEOL thermal budget, definitely combining BEOL process compatibility, performance reliability, low power programming and scalability demonstrated at array level.

\section{REFERENCES}

[1] H.-Y. Cheng, F. Carta, W.-C. Chien, H.-L. Lung, and M. J. BrightSky, "3D cross-point phase-change memory for storage-class memory," Journal of Physics D: Applied Physics, vol. 52, no. 47, p. 473002, 2019.

[2] F. Arnaud, P. Zuliani, J. Reynard, A. Gandolfo, F. Disegni, P. Mattavelli, E. Gomiero, G. Samanni, C. Jahan, R. Berthelon et al., "Truly innovative $28 \mathrm{~nm}$ FDSOI technology for automotive micro-controller applications embedding 16MB phase change memory," in 2018 IEEE International Electron Devices Meeting (IEDM). IEEE, 2018, pp. 18-4.

[3] F. Xiong, M.-H. Bae, Y. Dai, A. D. Liao, A. Behnam, E. A. Carrion, S. Hong, D. Ielmini, and E. Pop, "Self-aligned nanotube-nanowire phase change memory," Nano letters, vol. 13, no. 2, pp. 464-469, 2013.

[4] I. Kim, S. Cho, D. Im, E. Cho, D. Kim, G. Oh, D. Ahn, S. Park, S. Nam, J. Moon et al., "High performance PRAM cell scalable to sub-20nm technology with below $4 \mathrm{~F}^{2}$ cell size, extendable to DRAM applications," in 2010 Symposium on VLSI Technology. IEEE, 2010, pp. 203-204.

[5] H. Lung, Y. Ho, Y. Zhu, W. Chien, S. Kim, W. Kim, H. Cheng, A. Ray, M. Brightsky, R. Bruce et al., "A novel low power phase change memory using inter-granular switching," in 2016 IEEE Symposium on VLSI Technology. IEEE, 2016, pp. 1-2.

[6] C. Ahn, S. W. Fong, Y. Kim, S. Lee, A. Sood, C. M. Neumann, M. Asheghi, K. E. Goodson, E. Pop, and H.-S. P. Wong, "Energyefficient phase-change memory with graphene as a thermal barrier," Nano letters, vol. 15, no. 10, pp. 6809-6814, 2015.

[7] Q. Hubert, C. Jahan, A. Toffoli, L. Perniola, V. Sousa, A. Persico, J. Nodin, H. Grampeix, F. Aussenac, and B. De Salvo, "Reset current reduction in phase-change memory cell using a thin interfacial oxide layer," in 2011 Proceedings of the European Solid-State Device Research Conference (ESSDERC). IEEE, 2011, pp. 95-98.

[8] E. Gourvest, B. Pelissier, C. Vallée, A. Roule, S. Lhostis, and S. Maitrejean, "Impact of oxidation on $\mathrm{Ge}_{2} \mathrm{Sb}_{2} \mathrm{Te}_{5}$ and $\mathrm{GeTe}$ phase-change properties," Journal of The Electrochemical Society, vol. 159, no. 4, p. H373, 2012.

[9] R. Berthier, N. Bernier, D. Cooper, C. Sabbione, F. Hippert, and P. Noé, "In situ observation of the impact of surface oxidation on the crystallization mechanism of GeTe phase-change thin films by scanning transmission electron microscopy," Journal of Applied Physics, vol. 122, no. 11, p. $115304,2017$.

[10] S. Hanzawa, N. Kitai, K. Osada, A. Kotabe, Y. Matsui, N. Matsuzaki, N. Takaura, M. Moniwa, and T. Kawahara, "A $512 \mathrm{kB}$ embedded phase change memory with $416 \mathrm{kB} / \mathrm{s}$ write throughput at $100 \mu \mathrm{A}$ cell write current," in 2007 IEEE International Solid-State Circuits Conference. Digest of Technical Papers. IEEE, 2007, pp. 474-616.

[11] Y. Choi, I. Song, M.-H. Park, H. Chung, S. Chang, B. Cho, J. Kim, Y. Oh, D. Kwon, J. Sunwoo et al., "A 20nm 1.8V 8Gb PCRAM with $40 \mathrm{MB} / \mathrm{s}$ program bandwidth," in 2012 IEEE International Solid-State Circuits Conference. IEEE, 2012, pp. 46-48.

[12] E. S. Gedraite, "Analysis of the effect of electrode shapes in dielectric breakdown of thin films and prevision of breakdown using partial discharges." 
[13] D. Ielmini and Y. Zhang, "Analytical model for subthreshold conduction and threshold switching in chalcogenide-based memory devices," Journal of Applied Physics, vol. 102, no. 5, p. 054517, 2007.

[14] S. Lee, J.-h. Jeong, T. S. Lee, W. M. Kim, and B.-k. Cheong, "A study on the failure mechanism of a phase-change memory in write/erase cycling," IEEE Electron Device Letters, vol. 30, no. 5, pp. 448-450, 2009.

[15] I. Chambouleyron and A. Zanatta, "Nitrogen in germanium," Journal of applied physics, vol. 84, no. 1, pp. 1-30, 1998.

[16] O. Cueto, V. Sousa, G. Navarro, and S. Blonkowski, "Coupling the phase-field method with an electrothermal solver to simulate phase change mechanisms in PCRAM cells," in 2015 International Conference on Simulation of Semiconductor Processes and Devices (SISPAD). IEEE, 2015, pp. 301-304. 